\title{
UNICRITICAL POLYNOMIAL MAPS WITH RATIONAL MULTIPLIERS
}

\begin{abstract}
VALENTIN HUGUIN
Abstract. In this article, we prove that every unicritical polynomial map that has only rational multipliers is either a power map or a Chebyshev map. This provides some evidence in support of a conjecture by Milnor concerning rational maps whose multipliers are all integers.
\end{abstract}

\section{INTRODUCTION}

Given a polynomial map $f: \mathbb{C} \rightarrow \mathbb{C}$ and a point $z_{0} \in \mathbb{C}$, we study the sequence $\left(f^{\circ n}\left(z_{0}\right)\right)_{n \geq 0}$ of iterates of $f$ at $z_{0}$. The set $\left\{f^{\circ n}\left(z_{0}\right): n \geq 0\right\}$ is called the forward orbit of $z_{0}$ under $f$.

The point $z_{0}$ is said to be periodic for $f$ if there exists an integer $n \geq 1$ such that $f^{\circ n}\left(z_{0}\right)=z_{0}$; the least such integer $n$ is called the period of $z_{0}$. The forward orbit of $z_{0}$, which has cardinality $n$, is said to be a cycle for $f$. The multiplier of $f$ at $z_{0}$ is the derivative of $f^{\circ n}$ at $z_{0}$; equivalently, it is the product of the derivatives of $f$ along the cycle. In particular, $f$ has the same multiplier at each point of the cycle.

The multiplier is invariant under conjugacy: if $f$ and $g$ are two polynomial maps, $\phi$ is an invertible affine map such that $\phi \circ f=g \circ \phi$ and $z_{0}$ is a periodic point for $f$, then $\phi\left(z_{0}\right)$ is a periodic point for $g$ with the same period and the same multiplier.

In this paper, we wish to examine the polynomial maps that have only integer or rational - multipliers.

Definition 1. A polynomial map $f: \mathbb{C} \rightarrow \mathbb{C}$ of degree $d \geq 2$ is said to be a power map if it is affinely conjugate to $z \mapsto z^{d}$.

For every $d \geq 2$, there exists a unique polynomial $T_{d} \in \mathbb{C}[z]$ such that

$$
T_{d}\left(z+z^{-1}\right)=z^{d}+z^{-d} \text {. }
$$

The polynomial $T_{d}$ is monic of degree $d$ and is called the $d$ th Chebyshev polynomial.

Example 2. We have $T_{2}(z)=z^{2}-2$ and $T_{3}(z)=z^{3}-3 z$.

Definition 3. A polynomial map $f: \mathbb{C} \rightarrow \mathbb{C}$ of degree $d \geq 2$ is said to be a Chebyshev map if it is affinely conjugate to $\pm T_{d}$.

Remark 4 . For every $d \geq 2$, the polynomials $-T_{d}$ and $T_{d}$ are affinely conjugate if and only if $d$ is even.

These conjugacy classes of polynomials share the following well-known property:

Proposition 5 ([Mil06, Corollary 3.9]). Suppose that $f: \mathbb{C} \rightarrow \mathbb{C}$ is a power map or a Chebyshev map. Then $f$ has only integer multipliers.

Received by the editors November 18, 2020, and, in revised form, April 29, 2021, and May 17, 2021.

2020 Mathematics Subject Classification. Primary 37P05, 37P35; Secondary 37F10, 37F44. 
1.1. Statement of the results. We are interested in the converse of Proposition 5] More precisely, we wish to show that every polynomial map that has only integer or rational - multipliers is either a power map or a Chebyshev map.

We restrict ourselves to unicritical polynomial maps - that is, polynomial maps of degree $d \geq 2$ that have a unique critical point in the complex plane.

Theorem 6. Assume that $f: \mathbb{C} \rightarrow \mathbb{C}$ is a unicritical polynomial map that has only rational multipliers. Then $f$ is either a power map or a Chebyshev map.

Remark 7 . For every $d \geq 2$, the polynomial $T_{d}$ has exactly $d-1$ critical points given by $2 \cos \left(\frac{\pi j}{d}\right)$ for $j \in\{1, \ldots, d-1\}$. In particular, a Chebyshev map is unicritical if and only if it has degree 2 .

Using similar arguments, we also obtain a result concerning cubic polynomial maps with symmetries - that is, cubic polynomial maps that commute with a nontrivial invertible affine map.

Theorem 8. Assume that $f: \mathbb{C} \rightarrow \mathbb{C}$ is a cubic polynomial map with symmetries that has only integer multipliers. Then $f$ is a power map or a Chebyshev map.

1.2. Motivation. In a more general setting, Milnor conjectured in Mil06 that power maps, Chebyshev maps and flexible Lattès maps are the only rational maps whose multipliers are all integers. We may even extend his question as follows:

Question 9. Let $K$ be a number field, and denote by $\mathcal{O}_{K}$ its ring of integers. Assume that $f: \widehat{\mathbb{C}} \rightarrow \widehat{\mathbb{C}}$ is a rational map whose multipliers all lie in $\mathcal{O}_{K}-$ or $K$. Is $f$ necessarily a finite quotient of an affine map - that is, either a power map, a Chebyshev map or a Lattès map?

We give here a positive answer in the case of rational numbers and unicritical polynomial maps. To the author's knowledge, this question has not been studied before. This one can be viewed as an analog of questions concerning rational preperiodic points for a rational map, which have received a lot of attention (see $\mathrm{BIJ}^{+} 19$. and [Sil07]).

In EvS11, Eremenko and van Strien investigated the rational maps that have only real multipliers: they proved that, if $f: \widehat{\mathbb{C}} \rightarrow \widehat{\mathbb{C}}$ is such a map, then either $f$ is a Lattès map or its Julia set $\mathcal{J}_{f}$ is contained in a circle; they also gave a description of these maps.

\section{Proofs of the Results}

We shall prove here Theorem 6 and Theorem 8 , Our proofs rely on the result below, which states that our problem comes down to examining the factorizations of certain polynomials related to the multipliers of a monic polynomial.

Fix an integer $d \geq 2$. Since every polynomial map $f: \mathbb{C} \rightarrow \mathbb{C}$ is affinely conjugate to a monic polynomial map and the multiplier is invariant under conjugacy, we may restrict our attention to monic polynomials.

Proposition 10. Assume that $f: \mathbb{C} \rightarrow \mathbb{C}$ is a monic polynomial map of degree $d$. Then

(1) there exists a unique sequence $\left(\Phi_{n}^{f}\right)_{n \geq 1}$ of elements of $\mathbb{C}[z]$ such that, for every $n \geq 1$, we have

$$
f^{\circ n}(z)-z=\prod_{k \mid n} \Phi_{k}^{f}(z) ;
$$


(2) for every $n \geq 1$, there is a unique monic polynomial $M_{n}^{f} \in \mathbb{C}[\lambda]$ such that

$$
M_{n}^{f}(\lambda)^{n}=\operatorname{res}_{z}\left(\Phi_{n}^{f}(z), \lambda-\left(f^{\circ n}\right)^{\prime}(z)\right),
$$

where res $_{z}$ denotes the resultant with respect to $z$;

(3) given a subring $R$ of $\mathbb{C}$ and $n \geq 1$, the multipliers of $f$ at its cycles with period $n$ all lie in $R$ if and only if $M_{n}^{f}$ splits into linear factors of $R[\lambda]$.

Definition 11. Suppose that $f: \mathbb{C} \rightarrow \mathbb{C}$ is a monic polynomial map of degree $d$. For $n \geq 1$, the polynomial $\Phi_{n}^{f}$ is called the $n$th dynatomic polynomial of $f$ and the polynomial $M_{n}^{f}$ is called the $n$th multiplier polynomial of $f$.

Proof of Proposition 10, For a proof of (10), we refer the reader to [MP94, Section 2] or [VH92, Section 2]). For a proof of (2), see [MP94, Section 5] or [VH92, Section 4]. Now, suppose that $n \geq 1$. Then we have

$$
M_{n}^{f}(\lambda)^{n}=\prod_{j=1}^{r}\left(\lambda-\left(f^{\circ n}\right)^{\prime}\left(z_{j}\right)\right),
$$

where $z_{1}, \ldots, z_{r}$ are the - not necessarily distinct - roots of the polynomial $\Phi_{n}^{f}$. By [MS95, Proposition 3.2], a point $z_{0} \in \mathbb{C}$ is a root of $\Phi_{n}^{f}$ if and only if either $z_{0}$ is a periodic point for $f$ with period $n$ or $z_{0}$ is a periodic point for $f$ with period a proper divisor $k$ of $n$ and multiplier a primitive $\frac{n}{k}$ th root of unity. Note that, if $z_{0} \in \mathbb{C}$ is a periodic point for $f$ with period a divisor $k$ of $n$ and multiplier a $\frac{n}{k}$ th root of unity, then we have $\left(f^{\circ n}\right)^{\prime}\left(z_{0}\right)=1$ by the chain rule. This completes the proof of (3).

For $c \in \mathbb{C}$, let $f_{c}: \mathbb{C} \rightarrow \mathbb{C}$ be the polynomial map

$$
f_{c}: z \mapsto z^{d}+c .
$$

For every $c \in \mathbb{C}$, the map $f_{c}$ is unicritical with critical point 0 and critical value $c$. Furthermore, if $f: \mathbb{C} \rightarrow \mathbb{C}$ is a unicritical polynomial map of degree $d$, then there exists a parameter $c \in \mathbb{C}$ - which is unique up to multiplication by a $(d-1)$ th root of unity - such that $f$ is affinely conjugate to $f_{c}$.

Consequently, to prove Theorem $[$, we are reduced to determining the parameters $c \in \mathbb{C}$ for which the polynomials $M_{n}^{f_{c}} \in \mathbb{C}[\lambda]$, with $n \geq 1$, have only rational roots. Note that, if $c \in \mathbb{C}$ is such a parameter, then, for every $n \geq 1$, the polynomial $M_{n}^{f_{c}}$ lies in $\mathbb{Q}[\lambda]$ and its discriminant

$$
\Delta_{n}(c)=\operatorname{disc} M_{n}^{f_{c}}
$$

is the square of a rational number. In fact, we shall see that, to prove Theorem 6, it suffices to examine the polynomials $M_{n}^{f_{c}}$ for only a few small values of $n$.

2.1. Quadratic polynomial maps. Let us examine here the quadratic polynomials that have only integer - or rational - multipliers.

Suppose that $d=2$. Then, for every $c \in \mathbb{C}$, the map $f_{c}$ is a power map if and only if $c=0$ and is a Chebyshev map if and only if $c=-2$. Using the software SageMath, we can compute $M_{n}^{f_{c}}$ and $\Delta_{n}(c)$ for $c \in \mathbb{C}$ and small values of $n$. 
Example 12. For every $c \in \mathbb{C}$, we have

$$
\begin{aligned}
M_{1}^{f_{c}}(\lambda)= & \lambda^{2}-2 \lambda+4 c, \\
M_{2}^{f_{c}}(\lambda)= & \lambda-4 c-4, \\
M_{3}^{f_{c}}(\lambda)= & \lambda^{2}+(-8 c-16) \lambda+64 c^{3}+128 c^{2}+64 c+64, \\
M_{4}^{f_{c}}(\lambda)= & \lambda^{3}+\left(16 c^{2}-48\right) \lambda^{2}+\left(-256 c^{4}-256 c^{3}+256 c^{2}+768\right) \lambda \\
& -4096 c^{6}-12288 c^{5}-12288 c^{4}-12288 c^{3}-8192 c^{2}-4096 .
\end{aligned}
$$

Remark 13. For every $n \geq 1$, the coefficients of $M_{n}^{f_{c}}$ are polynomials in $4 c$ with integer coefficients (see [Bou14, Lemma 1] or Hug21]).

Example 14. For every $c \in \mathbb{C}$, we have

$$
\begin{aligned}
& \Delta_{1}(c)=-2^{2}(4 c-1), \\
& \Delta_{2}(c)=1, \\
& \Delta_{3}(c)=-2^{6}(4 c+7) c^{2}, \\
& \Delta_{4}(c)=-2^{24}\left(64 c^{3}+144 c^{2}+108 c+135\right)(c+2)^{2} c^{6} .
\end{aligned}
$$

Remark 15. It follows from [Mor96, Proposition 9] that, for every $n \geq 1$, the roots of $\Delta_{n}$ that have an odd multiplicity are precisely the parameters $c_{0} \in \mathbb{C}$ for which the map $f_{c_{0}}$ has a cycle with period $n$ and multiplier 1 .

First, let us examine the quadratic polynomials whose multipliers are integers. By Proposition 10, for every $c \in \mathbb{C}$, the map $f_{c}$ has an integer multiplier at each cycle with period 1 or 2 if and only if the polynomials $M_{1}^{f_{c}}$ and $M_{2}^{f_{c}}$ split into linear factors of $\mathbb{Z}[\lambda]$, which occurs if and only if there exists $m \in \mathbb{Z}$ such that $c=\frac{1-m^{2}}{4}$. In particular, there exist infinitely many such parameters $c \in \mathbb{C}$. In contrast, by considering also the multipliers at the cycles with period 3 , we obtain the following:

Proposition 16. Assume that $f: \mathbb{C} \rightarrow \mathbb{C}$ is a quadratic polynomial map that has an integer multiplier at each cycle with period less than or equal to 3 . Then $f$ is either a power map or a Chebyshev map.

Proof. There exists a parameter $c \in \mathbb{C}$ such that $f$ is affinely conjugate to $f_{c}$. By Proposition 10, the polynomials $M_{n}^{f_{c}}$, with $n \in\{1,2,3\}$, split into linear factors of $\mathbb{Z}[\lambda]$, and hence $4 c$ is an integer and

$$
\Delta_{1}(c)=-2^{2}(4 c-1) \quad \text { and } \quad \Delta_{3}(c)=-2^{6}(4 c+7) c^{2}
$$

are the squares of integers. Therefore, either $c=0$ or there exist $a, b \in \mathbb{Z}_{\geq 0}$ such that

$$
-(4 c-1)=a^{2} \quad \text { and } \quad-(4 c+7)=b^{2} .
$$

In the latter case, we have $(a-b)(a+b)=8$, and hence

$$
\left\{\begin{array} { l } 
{ a - b = 1 } \\
{ a + b = 8 }
\end{array} \text { or } \left\{\begin{array}{l}
a-b=2 \\
a+b=4
\end{array},\right.\right.
$$

which yields $(a, b)=(3,1)$ and $c=-2$. Thus, the proposition is proved.

Let us now study the quadratic polynomial maps whose multipliers are rational. There exist infinitely many parameters $c \in \mathbb{C}$ for which the map $f_{c}$ has a rational multiplier at each cycle with period less than or equal to 3 . More precisely, a 
parameter $c \in \mathbb{C}$ has this property if and only if $c$ is rational and $\Delta_{1}(c)$ and $\Delta_{3}(c)$ are the squares of rational numbers, which occurs if and only if $c=0$ or there exists $r \in \mathbb{Q}_{\neq 0}$ such that $c=\frac{-\left(r^{4}+3 r^{2}+4\right)}{4 r^{2}}$. In contrast, by considering also the multipliers at the cycles with period 4 , we are led to examine the rational points on a certain elliptic curve and we obtain the following result, which is a stronger version of Theorem 6 in the case of quadratic polynomials:

Proposition 17. Assume that $f: \mathbb{C} \rightarrow \mathbb{C}$ is a quadratic polynomial map that has a rational multiplier at each cycle with period less than or equal to 4 . Then $f$ is either a power map or a Chebyshev map.

Proof. There exists a parameter $c \in \mathbb{C}$ such that $f$ is affinely conjugate to $f_{c}$. By Proposition 10, the polynomials $M_{n}^{f_{c}}$, with $n \in\{1, \ldots, 4\}$, split into linear factors of $\mathbb{Q}[\lambda]$, and hence $c$ is rational and

$$
\Delta_{4}(c)=-2^{24}\left(64 c^{3}+144 c^{2}+108 c+135\right)(c+2)^{2} c^{6}
$$

is the square of a rational number. Note that, if there exists $r \in \mathbb{Q}$ such that

$$
-\left(64 c^{3}+144 c^{2}+108 c+135\right)=r^{2},
$$

then we have $r^{2}=(-4 c-3)^{3}-108$, which contradicts the fact that the group of rational points of the elliptic curve defined by $y^{2}=x^{3}-108$ is trivial (see [LMF20]). Therefore, we have $c \in\{-2,0\}$. Thus, the proposition is proved.

Remark 18. By a change of variables, proving that the elliptic curve appearing in the proof of Proposition 17 has a trivial group of rational points is equivalent to showing that the Diophantine equation $x^{3}+y^{3}=4 z^{3}$ has no solution $(x, y, z) \in \mathbb{Z}^{3}$ with $z \neq 0$. This latter fact can be proved by infinite descent (see Hug21]).

Remark 19. It follows from [EvS11, Theorem 1] that, for every $c \in \mathbb{C}$, the map $f_{c}$ has a real multiplier at each cycle if and only if $c \in(-\infty,-2] \cup\{0\}$. In particular, the property of having only real multipliers does not characterize power maps and Chebyshev maps among the quadratic polynomials.

2.2. Unicritical polynomial maps of degree at least 3. We shall see here that, unlike in the case of quadratic polynomials, power maps are the only unicritical polynomial maps of degree at least 3 that have only real multipliers. Note that, for every $c \in \mathbb{C}$, the map $f_{c}$ is a power map if and only if $c=0$.

First, suppose that $d=3$. Using the software SageMath, we can compute $M_{n}^{f_{c}}$ and $\Delta_{n}(c)$ for $c \in \mathbb{C}$ and $n \in\{1,2\}$.

Example 20. For every $c \in \mathbb{C}$, we have

$$
M_{1}^{f_{c}}(\lambda)=\lambda^{3}-6 \lambda^{2}+9 \lambda-27 c^{2}
$$

and

$$
M_{2}^{f_{c}}(\lambda)=\lambda^{3}-27 \lambda^{2}+\left(162 c^{2}+243\right) \lambda-729 c^{4}-1458 c^{2}-729 .
$$

Example 21. For every $c \in \mathbb{C}$, we have

$$
\Delta_{1}(c)=-3^{6}\left(27 c^{2}-4\right) c^{2} \quad \text { and } \quad \Delta_{2}(c)=-3^{12}\left(27 c^{2}+32\right) c^{6} .
$$

It follows from Proposition 10 that, for every $c \in \mathbb{C}$, the map $f_{c}$ has a real multiplier at each fixed point if and only if $c^{2}$ is real and $\Delta_{1}(c) \geq 0$, which occurs if and only if $c^{2} \in\left[0, \frac{4}{27}\right]$. In particular, power maps are not the only cubic unicritical polynomial maps whose multiplier at each fixed point is real. 
Remark 22. There also exist infinitely many parameters $c \in \mathbb{C}$ for which the map $f_{c}$ has a rational multiplier at each fixed point. More precisely, a parameter $c \in \mathbb{C}$ has this property if and only if the polynomial $M_{1}^{f_{c}}$ has a rational root and its discriminant $\Delta_{1}(c)$ is the square of a rational number, which occurs if and only if there exists $r \in \mathbb{Q}$ such that $c^{2}=\frac{4\left(r^{2}-1\right)^{2}}{\left(r^{2}+3\right)^{3}}$.

In contrast, by considering also the multipliers at the cycles with period 2 , we obtain the result below, which immediately implies Theorem 6 in the case of cubic unicritical polynomials.

Proposition 23. Assume that $f: \mathbb{C} \rightarrow \mathbb{C}$ is a cubic unicritical polynomial map that has a real multiplier at each cycle with period 1 or 2 . Then $f$ is a power map.

Proof. There exists a parameter $c \in \mathbb{C}$ such that $f$ is affinely conjugate to $f_{c}$. By Proposition 10, the polynomials $M_{1}^{f_{c}}$ and $M_{2}^{f_{c}}$ split into linear factors of $\mathbb{R}[\lambda]$, and hence $c^{2}$ is real and

$$
\Delta_{1}(c)=-3^{6}\left(27 c^{2}-4\right) c^{2} \geq 0 \quad \text { and } \quad \Delta_{2}(c)=-3^{12}\left(27 c^{2}+32\right) c^{6} \geq 0 .
$$

Therefore, we have

$$
c^{2} \in\left[\frac{-32}{27}, 0\right] \cap\left[0, \frac{4}{27}\right]=\{0\} .
$$

Thus, the proposition is proved.

Let us now investigate the unicritical polynomial maps of degree at least 4 whose multipliers are real. We shall see that, unlike in the case of cubic unicritical polynomials, the property of having a real multiplier at each fixed point characterizes here power maps. Our result relies on the calculation of $M_{1}^{f_{c}}$ for $c \in \mathbb{C}$.

Example 24. Suppose that $d \geq 2$ and $c \in \mathbb{C}$. Then we have

$$
M_{1}^{f_{c}}(\lambda)=\operatorname{res}_{z}\left(z^{d}-z+c, \lambda-d z^{d-1}\right)=(-d)^{d} \prod_{j=1}^{d-1}\left(z_{j}^{d}-z_{j}+c\right),
$$

where $z_{1}, \ldots, z_{d-1}$ are the roots of $d z^{d-1}-\lambda \in \mathbb{C}[z]$. It follows that

$$
\begin{aligned}
M_{1}^{f_{c}}(\lambda) & =(-d)^{d} \prod_{j=1}^{d-1}\left(d^{-1}(\lambda-d) z_{j}+c\right) \\
& =(-d)^{d}\left(c^{d-1}+\sum_{j=1}^{d-1} d^{-j}(\lambda-d)^{j} \sigma_{j} c^{d-1-j}\right),
\end{aligned}
$$

where $\sigma_{1}, \ldots, \sigma_{d-1}$ are the elementary symmetric functions of $z_{1}, \ldots, z_{d-1}$. Therefore, by the relations between roots and coefficients of a polynomial, we have

$$
M_{1}^{f_{c}}(\lambda)=\lambda(\lambda-d)^{d-1}+(-d)^{d} c^{d-1} .
$$

Remark 25. For every $n \geq 1$, the coefficients of $M_{n}^{f_{c}}$ are polynomials in $d^{d} c^{d-1}$ with integer coefficients (see Hug21] and compare [Mil14, Theorem 2.1]).

The following result is a stronger version of Theorem 6 in the case of unicritical polynomials of degree at least 4 .

Proposition 26. Assume that $f: \mathbb{C} \rightarrow \mathbb{C}$ is a unicritical polynomial map of degree $d \geq 4$ that has a real multiplier at each fixed point. Then $f$ is a power map. 
Proof. There exists a parameter $c \in \mathbb{C}$ such that $f$ is affinely conjugate to $f_{c}$. By Proposition 10, the polynomial

$$
M_{1}^{f_{c}}(\lambda)=\lambda(\lambda-d)^{d-1}+(-d)^{d} c^{d-1}
$$

splits into linear factors of $\mathbb{R}[\lambda]$, and hence the same is true of the polynomial

$$
L(\lambda)=\lambda^{d} M_{1}^{f_{c}}\left(\lambda^{-1}+d\right)=(-d)^{d} c^{d-1} \lambda^{d}+d \lambda+1
$$

and, by Rolle's theorem, of its derivative

$$
L^{\prime}(\lambda)=(-1)^{d} d^{d+1} c^{d-1} \lambda^{d-1}+d .
$$

Therefore, we have $c=0$ since the set of roots of $L^{\prime}$ is invariant under multiplication by a $(d-1)$ th root of unity and $d \geq 4$. Thus, the proposition is proved.

Finally, we have proved Theorem 6, which follows immediately from Proposition 17. Proposition 23 and Proposition 26.

2.3. Cubic polynomial maps with symmetries. We shall use here the same strategy to study the cubic polynomial maps with symmetries whose multipliers are integers and prove Theorem 8 .

For $a \in \mathbb{C}$, let $g_{a}: \mathbb{C} \rightarrow \mathbb{C}$ be the cubic polynomial map

$$
g_{a}: z \mapsto z^{3}+a z .
$$

For every $a \in \mathbb{C}$, the map $g_{a}$ fixes 0 with multiplier $a$ and commutes with $z \mapsto-z$. Furthermore, if $f: \mathbb{C} \rightarrow \mathbb{C}$ is a cubic polynomial map with symmetries, then there exists a unique parameter $a \in \mathbb{C}$ such that $f$ is affinely conjugate to $g_{a}$.

Unlike the family of cubic unicritical polynomial maps, the family of cubic polynomial maps with symmetries contains both power maps and Chebyshev maps. More precisely, for every $a \in \mathbb{C}$, the map $g_{a}$ is a power map if and only if $a=0$ and is a Chebyshev map if and only if $a= \pm 3$.

Using the software SageMath, we can compute $M_{n}^{g_{a}}$ for $a \in \mathbb{C}$ and $n \in\{1,2,3\}$.

Example 27. For every $a \in \mathbb{C}$, we have

$$
\begin{aligned}
& M_{1}^{g_{a}}(\lambda)=(\lambda-a)(\lambda+2 a-3)^{2}, \\
& M_{2}^{g_{a}}(\lambda)=\left(\lambda-4 a^{2}-12 a-9\right)\left(\lambda+2 a^{2}-9\right)^{2}, \\
& M_{3}^{g_{a}}(\lambda)=N_{3}(a, \lambda)^{2},
\end{aligned}
$$

where $N_{3} \in \mathbb{Z}[a, \lambda]$ is given by

$$
\begin{aligned}
N_{3}(a, \lambda)= & \lambda^{4}+\left(2 a^{3}+12 a^{2}-18 a-108\right) \lambda^{3} \\
& +\left(-48 a^{6}-72 a^{5}+396 a^{4}+486 a^{3}-324 a^{2}+1458 a+4374\right) \lambda^{2} \\
& +\left(32 a^{9}-792 a^{7}-432 a^{6}+5832 a^{5}+5832 a^{4}-7290 a^{3}-8748 a^{2}\right. \\
& -39366 a-78732) \lambda+256 a^{12}+384 a^{11}-4608 a^{10}-6912 a^{9} \\
& +24624 a^{8}+36936 a^{7}-23328 a^{6}-34992 a^{5}-131220 a^{4} \\
& -196830 a^{3}+236196 a^{2}+354294 a+531441 .
\end{aligned}
$$

Moreover, we have

$$
\operatorname{disc}_{\lambda} N_{3}(a, \lambda)=2^{12} 3^{12} D_{3}(a)\left(4 a^{3}+12 a^{2}-3 a-27\right)^{2}(a-3)^{4}(a+3)^{4} a^{12},
$$


where $\operatorname{disc}_{\lambda}$ denotes the discriminant with respect to $\lambda$ and $D_{3} \in \mathbb{Z}[a]$ is given by

$$
D_{3}(a)=4 a^{8}+16 a^{7}-35 a^{6}-206 a^{5}-113 a^{4}+376 a^{3}+715 a^{2}+1690 a+2197 .
$$

It follows from Proposition 10 that, for every $a \in \mathbb{C}$, the map $g_{a}$ has an integer multiplier at each cycle with period 1 or 2 if and only if $a$ is an integer. By considering also the multipliers at the cycles with period 3 , we obtain the following stronger version of Theorem 8

Proposition 28. Assume that $f: \mathbb{C} \rightarrow \mathbb{C}$ is a cubic polynomial map with symmetries that has an integer multiplier at each cycle with period less than or equal to 3. Then $f$ is either a power map or a Chebyshev map.

Proof. There exists a parameter $a \in \mathbb{C}$ such that $f$ is affinely conjugate to $g_{a}$. By Proposition 10, the polynomials $M_{n}^{g_{a}}$, with $n \in\{1,2,3\}$, split into linear factors of $\mathbb{Z}[\lambda]$, and hence $a$ is an integer and

$$
\operatorname{disc}_{\lambda} N_{3}(a, \lambda)=2^{12} 3^{12} D_{3}(a)\left(4 a^{3}+12 a^{2}-3 a-27\right)^{2}(a-3)^{4}(a+3)^{4} a^{12}
$$

is the square of an integer. Now, note that, if

$$
D_{3}(a)=4 a^{8}+16 a^{7}-35 a^{6}-206 a^{5}-113 a^{4}+376 a^{3}+715 a^{2}+1690 a+2197
$$

is the square of an integer, then its residue class in $\mathbb{Z} / 32 \mathbb{Z}$ is a square, and hence $a \equiv 1(\bmod 8)$. Moreover, observe that $D_{3}(1+8 b)$ is not the square of an integer whenever $b \in\{-7, \ldots, 13\}$ and we have

$$
L(b)^{2}<D_{3}(1+8 b)<(L(b)+1)^{2}
$$

for all $b \in \mathbb{Z} \backslash\{-7, \ldots, 13\}$, where

$$
L(b)=8192 b^{4}+6144 b^{3}+720 b^{2}-252 b-50 .
$$

Therefore, $D_{3}(a)$ is not the square of an integer, and hence $a \in\{-3,0,3\}$. Thus, the proposition is proved.

Using the software SageMath, we obtain that $D_{3}(a)$ is not the square of a rational number whenever $a$ is a rational number with height at most $10^{4}$. Thus, it seems likely that the question below has a negative answer, which would imply that every cubic polynomial map with symmetries that has a rational multiplier at each cycle with period less than or equal to 3 is either a power map or a Chebyshev map.

Question 29. Does the hyperelliptic curve of genus 3 over $\mathbb{Q}$ given by $b^{2}=D_{3}(a)$ have a rational point other than the two points at infinity?

Remark 30. Note that the curve of genus 1 given by $N_{3}(a, \lambda)=0$ together with the point $\left(\frac{9}{2}, \frac{1647}{4}\right)$ defines an elliptic curve $E$ over $\mathbb{Q}$. Using the software Magma, we obtain that its group of rational points $E(\mathbb{Q})$ is a free abelian group of rank 1. In particular, there exist infinitely many parameters $a \in \mathbb{C}$ for which the map $g_{a}$ has a rational multiplier at each cycle with period 1 or 2 and at a cycle with period 3. Another approach to proving that power maps and Chebyshev maps are the only cubic polynomial maps with symmetries that have a rational multiplier at each cycle with period less than or equal to 3 could be to show that the group $E(\mathbb{Q})$ does not contain 4 distinct points with the same $a$-coordinate. 


\section{ACKNOWLEDGMENTS}

The author would like to thank his Ph.D. advisors, Xavier Buff and Jasmin Raissy, for all their suggestions and encouragements.

\section{REFERENCES}

$\left[\mathrm{BIJ}^{+}\right.$19] Robert Benedetto, Patrick Ingram, Rafe Jones, Michelle Manes, Joseph H. Silverman, and Thomas J. Tucker, Current trends and open problems in arithmetic dynamics, Bull. Amer. Math. Soc. (N.S.) 56 (2019), no. 4, 611-685, DOI 10.1090/bull/1665. MR 4007163

[Bou14] Thierry Bousch, Les racines des composantes hyperboliques de $M$ sont des quarts d'entiers algébriques (French, with English summary), Frontiers in complex dynamics, Princeton Math. Ser., vol. 51, Princeton Univ. Press, Princeton, NJ, 2014, pp. 25-26. MR.3289904

[EvS11] Alexandre Eremenko and Sebastian van Strien, Rational maps with real multipliers, Trans. Amer. Math. Soc. 363 (2011), no. 12, 6453-6463, DOI 10.1090/S0002-9947-201105308-0. MR2833563

[Hug21] Valentin Huguin, Étude algébrique des points périodiques et des multiplicateurs d'une fraction rationnelle, 2021, Thesis (Ph.D.)-Université Toulouse III - Paul Sabatier (in preparation).

[LMF20] The LMFDB Collaboration, The L-functions and modular forms database, elliptic curve with LMFDB label 108. https://www.lmfdb.org/EllipticCurve/Q/108/a/1, 2020.

[Mil06] John Milnor, On Lattès maps, Dynamics on the Riemann sphere, Eur. Math. Soc., Zürich, 2006, pp. 9-43, DOI 10.4171/011-1/1. MR2348953

[Mil14] John Milnor, Arithmetic of unicritical polynomial maps, Frontiers in complex dynamics, Princeton Math. Ser., vol. 51, Princeton Univ. Press, Princeton, NJ, 2014, pp. 15-24. MR 3289903

[Mor96] Patrick Morton, On certain algebraic curves related to polynomial maps, Compositio Math. 103 (1996), no. 3, 319-350. MR1414593

[MP94] Patrick Morton and Pratiksha Patel, The Galois theory of periodic points of polynomial maps, Proc. London Math. Soc. (3) 68 (1994), no. 2, 225-263, DOI 10.1112/plms/s368.2.225. MR 1253503

[MS95] Patrick Morton and Joseph H. Silverman, Periodic points, multiplicities, and dynamical units, J. Reine Angew. Math. 461 (1995), 81-122, DOI 10.1515/crll.1995.461.81. MR 1324210

[Sil07] Joseph H. Silverman, The arithmetic of dynamical systems, Graduate Texts in Mathematics, vol. 241, Springer, New York, 2007, DOI 10.1007/978-0-387-69904-2. MR2316407

[VH92] Franco Vivaldi and Spyros Hatjispyros, Galois theory of periodic orbits of rational maps, Nonlinearity 5 (1992), no. 4, 961-978. MR.1174226

Institut de Mathématiques de Toulouse, UMR 5219, Université De Toulouse, CNRS, UPS, F-31062 Toulouse Cedex 9, France

Email address: valentin.huguin@math.univ-toulouse.fr 